\title{
Autoestima, autocompasión y afecto positivo y negativo en víctimas y agresores de bullying: Estudio comparativo con medidas autoinformadas e informadas por pares
}

\author{
Juan Múzquiz, Ana M. Pérez-García y José Bermúdez \\ Universidad Nacional de Educación a Distancia, Madrid, España
}

\begin{abstract}
Self-esteem, self-compassion and positive and negative affect in victims and bullies: A comparative study with self-report and peer-report measures
\end{abstract}

\begin{abstract}
Bullying is a prevalent problem with numerous negative consequences in psychological adjustment, especially for victims. However, most research on the topic is based on self-report measures subject to several biases and other limitations. The present study assessed self-esteem, positive and negative affect and self-compassion in 649 adolescents classified into victims, bullies, bully-victims and not involved students through peer- and self-report measures. Results showed that considering oneself a bully or a victim was associated with the psychological outcomes, being so in peer-report measures. Also, in selfreport measures, not involved participants showed higher levels of self-compassion than the other groups. The present research highlights the importance of including self-report and peer-report measures of bullying when studying the psychological adjustment of victims and bullies.
\end{abstract}

Keywords: Self-compassion; bullying; self-esteem; adolescence; affect; self-report; peer-report.

Resumen: El bullying es un problema altamente prevalente con numerosas consecuencias negativas sobre el ajuste psicológico, especialmente para las víctimas. Sin embargo, la mayoría de las investigaciones están basadas en autoinformes que están sujetos a diversos sesgos. La presente investigación evaluó la autoestima, el afecto positivo y negativo y la autocompasión de 649 adolescentes clasificados como víctimas, agresores, agresores-víctimas o no implicados utilizando medidas auto- y heteroinformadas por los compañeros. Los resultados mostraron que autorreconocerse como agresor o víctima guardó una asociación con las medidas de ajuste analizadas, más allá de serlo en las medidas heteroinformadas. En las medidas autoinformadas los participantes no implicados mostraron mayores niveles de autocompasión que los otros grupos. Se resalta la importancia de utilizar de manera conjunta medidas autoinformadas y heteroinformadas para estudiar variables relacionadas con el ajuste psicológico de víctimas y agresores.

Palabras clave: Autocompasión; bullying; autoestima; adolescencia; afecto; autoinforme; informe de pares.

\section{Introducción}

Un menor está sufriendo bullying (acoso entre iguales) cuando de manera frecuente y durante un periodo

Recibido: 02 de septiembre 2020; aceptado: 20 de octubre 2020 Correspondencia: Ana M. Pérez-García, Departamento de Personalidad, Evaluación y Tratamientos Psicológicos, Facultad de Psicología, Universidad Nacional de Educación a Distancia (UNED), C/ Juan del Rosal, 10, 28040 Madrid, España. Correo-e: aperez@psi.uned.es largo de tiempo está expuesto a las acciones negativas de otro compañero o grupo de compañeros que se encuentran en una posición de poder sobre la víctima en la que ésta no puede, o encuentra muy difícil defenderse por sí misma (Olweus, 1994). Los agresores pueden tener esta posición de poder por numerosas razones además de por la fuerza física: actuar en grupo, actuar con mayor crueldad, manejar mejor el idioma o estar más respaldado por los compañeros. Las acciones negativas son realizadas a propósito, con la intención de causar un daño y toman 
diversas formas: verbal (insultos, motes ofensivos) (Björkqvist et al., 1992); física (golpes, zancadillas, robos) (Olweus, 1994) o social que va encaminada a dañar el estatus de la víctima y provocar su aislamiento en el grupo de clase (p.ej., difusión de rumores) (Crick y Grotpeter, 1995). En un estudio reciente, Magaz et al. (2016) aislaron los siguientes tipos de bullying: físico, verbal, exclusión social (directa e indirecta), amenazas, ciberbullying, y agresión basada en objetos.

La prevalencia estimada del acoso varía ampliamente debido a las diferentes muestras y a la disparidad en la metodología utilizada (ver Solberg y Olweus, 2003). En un estudio internacional que empleó la misma metodología en 40 países, la prevalencia osciló entre el 8.6\% (Suecia) y más del 40\% (Lituania). En dicho estudio, la prevalencia en España se sitúo en torno al 9\%. También en España, con una amplia muestra, Díaz-Aguado Jalón et al. (2013) encontraron que un 3.8\% declaraba sufrirlo con frecuencia. Así mismo, basándose en una muestra de 600 adolescentes, Magaz et al. (2011), reportaron que el $34 \%$ de los participantes informaron haber sufrido «muchas veces» al menos una conducta de acoso. En resumen y, en el mejor de los casos, de media podría decirse que lo sufre al menos un alumno de cada clase de la Educación Secundaria Obligatoria en España.

Dada su alta prevalencia, sufrir acoso es un importante factor de riesgo para el desarrollo de problemas psicológicos y sintomatología psicopatológica tanto en la niñez/ adolescencia como en la edad adulta. Ser víctima de acoso ha sido asociado a bajo rendimiento escolar (Schwartz et al., 2005), ansiedad (Swearer et al., 2001), síntomas psicosomáticos como insomnio o dolor de cabeza (Natvig et al., 2001), sintomatología depresiva (Brunstein Klomek et al., 2019; Sweeting et al., 2006), mayor probabilidad de provocarse autolesiones e ideación suicida (Bang y Park, 2017; Obando et al., 2018; Pedreira-Massa, 2019), psicosis (Bang y Park, 2017), trastorno por atracón y bulimia (Lie et al., 2019) y trastorno por estrés postraumático (Houbre et al., 2006). Asimismo, las víctimas tienden a mostrar un peor ajuste emocional (Khatri et al., 2000) y una autoestima más baja especialmente en el terreno social (Estévez et al., 2009; Kowalski y Limber, 2013). Además, como Moore et al. (2017) recogen en su meta-análisis, el bullying en estudios longitudinales antecede en muchos casos al desarrollo de la sintomatología.

Las investigaciones sobre el ajuste psicológico de los agresores han arrojado resultados contradictorios. Algunas investigaciones han encontrado diversos problemas psicológicos entre los agresores como sintomatología depresiva (Moore et al., 2014), insatisfacción con la vida (MacDonald et al., 2005) y una mayor probabilidad de tener intentos autolíticos (Hinduja y Patchin, 2019). Sin embargo, otras investigaciones no han encontrado que los agresores muestren más sintomatología ansiosa o depresiva que los no implicados (no agresores) (Kowalski y Limber, 2013) o tengan un déficit en inteligencia social (Salmivalli et al., 1999). Además, pueden tener un buen concepto de sí mismos, una autoestima alta, al menos igual a los no implicados, y superior a las víctimas (Cerezo, 2009), un buen estatus y, en muchos casos, reciben admiración por parte del grupo que les hace sentirse bien (Rigby y Slee, 1991). El hecho de que los agresores obtengan iguales o incluso superiores puntuaciones en autoestima lleva a pensar si tener la autoestima «alta» es lo mismo que gozar de una buena salud mental, por lo que el concepto de autoestima como indicador indiscutible de salud mental ha sufrido críticas de varios autores (Baumeister et al., 2003; Leary y Baumeister, 2000; Neff, 2009).

Neff (2003) comenzó con el estudio de la autocompasión como un modo más saludable de relacionarnos con nosotros mismos, especialmente al enfrentarnos con momentos de dolor, fracaso o sufrimiento. La autocompasión implica la interrelación de tres componentes. En primer lugar, la bondad para con uno mismo frente a tratarse con crueldad ante los fallos e imperfecciones. En segundo lugar, el sentido de humanidad compartida, por el cual la persona entiende que sufrir y equivocarse forman parte de la condición humana, frente a los sentimientos de aislamiento, que sería dejarse llevar por pensamientos y sentimientos de que lo malo que le ocurre a uno es exclusivo de uno mismo. En tercer lugar, el componente mindfulness, que tiene que ver con la capacidad para experimentar las propias emociones con equilibrio, frente a la sobreidentificación, que sería dejarnos arrastrar por las emociones sin la capacidad para atenderlas con claridad y perspectiva. El concepto de autocompasión ha sido investigado tanto con una puntuación total, como a través de dos puntuaciones diferentes, una autocompasión positiva (self-warmth) que agruparía los aspectos positivos y otra que agruparía los aspectos negativos de la escala (self-coldness) (Gilbert et al., 2011; López et al., 2015). La autocompasión ha sido asociada positivamente con diversas variables psicológicas deseables como el bienestar emocional (Bluth y Blanton, 2015), o la inteligencia emocional (Castilho et al., 2017). Asimismo, se ha encontrado que la autocompasión puede ejercer como factor protector frente al estrés (Galla, 2016), los acontecimientos traumáticos (Zeller et al., 2015), la polivictimización (Játiva y Cerezo, 2014), la ideación suicida (Xavier et al., 2016) o el estigma (Vigna et al., 2018). La evidencia de la literatura también muestra que es posible incrementar los niveles de autocompasión y que esto mejore el bienestar y reduzca la sintomatología psicopatológica (Bluth y Neff, 2018; Edwards et al., 
2014). Por tanto, la autocompasión se propone como un importante recurso psicológico para afrontar las dificultades de la vida. Aunque se ha estudiado el papel mediador de la autocompasión frente a la polivictimización, todavía no se han estudiado los niveles diferenciales de autocompasión en función del rol en la dinámica del bullying.

Tanto los estudios de prevalencia como los estudios que han relacionado el acoso con la autoestima o el afecto están basados, en su gran mayoría, en medidas autoinformadas, donde el propio alumno informa sobre si lo sufre o lo ejerce; sin embargo, los participantes pueden estar sometidos a problemas como el miedo a la hora de responder o contestar sesgados por la deseabilidad social (Estévez et al., 2009; Solberg y Olweus, 2003). Varios artículos han recomendado el uso de medidas informadas por pares para aclarar estas cuestiones, pero los estudios que han hecho uso combinado de medidas auto y heteroinformadas son escasos (Vivolo-Kantor et al., 2014).

Por tanto, los objetivos del presente estudio fueron en primer lugar estudiar las posibles diferencias en autoestima, autocompasión y afecto (positivo y negativo) de agresores, víctimas y agresores-víctimas en la dinámica del bullying. En segundo lugar, investigar las diferencias en las medidas mencionadas anteriormente, en agresores y víctimas de acoso cuando estos comportamientos son registrados incluyendo medidas heteroinformadas. En tercer lugar, analizar las similitudes y diferencias de las variables autoestima y autocompasión en agresores y víctimas de acoso.

\section{Método}

\section{Participantes}

En el estudio participaron 649 alumnos de cuatro centros de Secundaria de la Comunidad de Madrid, dos de ellos públicos, uno concertado y uno privado. Las edades de los participantes estaban entre 12 y 17 años $(M=13.99 ; D T=1.20)$. Respecto al origen de los alumnos, la mayoría eran nacidos en España $(76.88 \%)$, el $11.03 \%$ eran de origen latinoamericano, el $6.04 \%$ europeos no españoles, el $2.41 \%$ africanos y el $1.82 \%$ asiáticos. Un $1.82 \%$ de los alumnos no contestaron a la pregunta sobre su lugar de nacimiento. Los menores no presentaban ninguna discapacidad psicológica o física que les impidiera contestar debidamente a las pruebas.

\section{Instrumentos}

Versión para adolescentes de la Escala PANAS de Afecto Positivo y Negativo (Sandín, 2003). Los alumnos debían responder en una escala Likert de 5 puntos $(0=$ Nada; $1=$ Un poco; $2=$ Bastante; $3=$ Mucho; $4=\mathrm{Mu}-$ chísimo) a la frecuencia con la que últimamente experimentaban 10 emociones positivas («siento que tengo vitalidad o energía», «me siento orgulloso/satisfecho») o 10 emociones negativas («tengo mal humor», «me siento culpable»), frente a los 3 puntos de la versión original (Nunca, A veces y Muchas veces) para igualarlo con otros formatos de respuesta de la investigación. La fiabilidad, medida mediante coeficiente alfa $(\alpha)$, en la muestra de este estudio para la escala de Afecto Negativo fue de .83 y de .77 para la escala de Afecto Positivo.

Escala de Autoestima de Rosenberg (Rosenberg, 1965; versión española de Echeburúa y Arbona, 1995). La escala se compone de 10 ítems, cinco de los cuales están formulados sobre aspectos positivos ( «Soy capaz de hacer las cosas tan bien como la mayoría de la gente») y 5 sobre aspectos negativos («En general, me inclino a pensar que soy un fracasado»). Los ítems se puntúan en una escala Likert de 4 puntos ( 1 = Muy en desacuerdo; 4 $=$ Muy de acuerdo). La fiabilidad de la escala en la presente muestra, medida por alfa de Cronbach $(\alpha)$, fue de .79 .

Escala de Autocompasión (Neff, 2003; versión española de Garcia-Campayo et al., 2014). Los 26 ítems de la escala incluyen 6 subescalas que pueden agruparse en dos puntuaciones: self-warmth (items positivos) y self-coldness (ítems negativos) (López et al., 2015). La escala se contesta en un formato Likert de 5 puntos que va de $1=$ casi nunca a $5=$ casi siempre. La fiabilidad $(\alpha)$ obtenida en la presente muestra fue de .79 para self-war$m t h, .85$ para self-coldness, y .84 para la autocompasión global.

Escala de Olweus (Olweus, 1996; versión española de Díaz-Aguado et al., 2013). Los alumnos completaron el ítem sobre frecuencia del acoso sufrido y ejercido del Cuestionario de Victimización de Olweus en el que se les presentó la definición de bullying y después, debían responder con qué frecuencia lo habían recibido o ejercido en los dos últimos meses $(1=$ nunca; $2=$ una o dos veces; 3 = prácticamente todas las semanas; $4=$ una vez a la semana por lo menos; 5 = varias veces cada semana).

Escalas heteroinformadas de bullying sufrido y ejercido (Babarro, 2014). Los participantes contestaron a 7 preguntas referentes a sus compañeros, sin límite de nominaciones. Tres de los ítems evaluaban el perfil de víctima («¿Qué compañero de clase es insultado o humillado por otros?») con una fiabilidad $(\alpha)$ de 0.90 . Los otros cuatro ítems evaluaban el perfil de agresor («¿Qué compañeros pegan o maltratan físicamente a otros?») y se obtuvo una fiabilidad $(\alpha)$ de .89 . 


\section{Procedimiento}

Los cuatro centros que participaron en el estudio contaron con la aprobación de la dirección o del Consejo Escolar. Tras aprobar su participación en el estudio, los propios centros mandaron una hoja de información y los menores aportaron el consentimiento informado de sus tutores legales o de los propios adolescentes, en función de la edad. La investigación contó también con la aprobación del Departamento de Personalidad, Evaluación y Tratamiento Psicológicos de la Universidad Nacional de Educación a Distancia. En todo momento se aseguró la confidencialidad y anonimato de los participantes. Las pruebas se pasaron en la clase de los alumnos, estando siempre presente un psicólogo para responder posibles dudas. La colaboración de los alumnos se premió con la participación en una lotería con entradas para el cine o el teatro. Las escuelas recibieron una formación para el profesorado y/o las familias sobre bullying y mejora de la convivencia.

Para la conformación de los distintos grupos se utilizó el siguiente procedimiento. Para el primer grupo de análisis, con medidas exclusivamente autoinformadas, se conformaron 4 grupos: agresores, víctimas, agresores-víctimas y grupo de control. Se consideraron víctimas aquellos que informaron de sufrir acoso con una frecuencia igual o superior a una o dos veces en los últimos 2 meses, adecuado para estimar las diferencias en ajuste psicológico (Solberg y Olweus, 2003).

Para los análisis que incluían medidas heteroinformadas, se conformaron cuatro grupos en el caso de las víctimas y otros cuatro en el caso de los agresores. En el caso de las víctimas quedaron los grupos: víctimas auto y heteroinformadas; víctimas solo heteroinformadas, víctimas solo autoinformadas y grupo de control (no víctimas). Para considerar a un alumno como una víctima heteroinformada, se realizó en primer lugar una suma de todas las puntuaciones obtenidas en la escala de víctima heteroinformada y se dividió por el número de alumnos presentes. Fueron consideradas víctimas las personas que obtuvieron más de una desviación típica en las puntuaciones seleccionadas para cada clase. Este procedimiento ya se ha seguido en otros estudios similares (Pellegrini et al., 1999; Solberg y Olweus, 2003). El mismo procedimiento se siguió para la clasificación de los agresores.

\section{Análisis estadístico}

Todos los datos fueron analizados con el programa SPSS.25. Para comparar las diferencias en función del género se realizaron tablas de contingencia utilizando el estadístico de $\chi^{2}$ y la $V$ de Cramer para estudiar la magnitud de las diferencias. Para la comparación entre grupos de víctimas, agresores y no implicados se realizó un análisis de varianza (ANOVA) y, en los casos en los que resultó significativo, se realizaron comparaciones entre los distintos grupos con la corrección de Bonferroni y se hizo uso del estadístico $d$ de Cohen para medir el tamaño del efecto de estas diferencias.

\section{Resultados}

\section{Prevalencia y datos descriptivos}

En primer lugar, se examinaron las prevalencias de acoso sufrido y ejercido en el total de la muestra. En el caso de las medidas autoinformadas, un $65.6 \%$ de los alumnos declararon no haber sufrido bullying en los dos últimos meses, un $27.4 \%$ informaron haber sufrido bullying una o dos ocasiones en los últimos dos meses, y un $7 \%$ declararon sufrirlo frecuentemente.

En cuanto al bullying ejercido, el $67.9 \%$ de los alumnos informaron de no haberlo practicado nunca, el $27.3 \%$ declararon haberlo ejercido alguna vez en los últimos dos meses y el $4.8 \%$ de los alumnos reconocieron haberlo ejercido con frecuencia. Para los siguientes análisis, se consideraron víctimas a los que lo habían sufrido con una frecuencia igual o superior a una o dos veces en los dos últimos meses, con lo que el grupo quedó conformado por el $34.4 \%$ de la muestra (Solberg y Olweus, 2003).

En las medidas heteroinformadas, y tras dicotomizar las variables, el $12.2 \%$ de los alumnos fue señalado por sus compañeros como posibles víctimas de acoso. El $13.9 \%$ de los alumnos fueron marcados por el resto de los compañeros como posibles agresores.

\section{Diferencias en función del género}

Las diferencias en función del género se muestran en la Tabla 1. Un porcentaje de chicos significativamente mayor informó llevar a cabo conductas de bullying con más frecuencia que las chicas; y también los chicos fueron señalados como agresores con más frecuencia por sus compañeros. En cualquier caso, el coeficiente $V$ de Cramer indica que estas diferencias fueron pequeñas. No se encontraron diferencias significativas en victimización ni en las medidas autoinformadas ni heteroinformadas. Sin embargo, sí se encontraron tendencias opuestas en función de si las medidas eran heteroinformadas o autoinformadas. Tomando en conjunto a las víctimas, un porcentaje mayor de chicos $(58.8 \%)$ fue señalado por sus compañeros como posibles víctimas en comparación 
con las chicas $(41.3 \%)\left(\chi^{2}=6.98 ; p<.01 ; V=.16\right)$. Sin embargo, un porcentaje mayor de chicas $(56.4 \%)$ decla- ró sufrir acoso respecto a los chicos $(43.6 \%)\left(\chi^{2}=3.25\right.$; $p=.07 ; V=.11)$

Tabla 1. Diferencias en las variables examinadas dependiendo del género

\begin{tabular}{|c|c|c|c|c|c|}
\hline & & $\begin{array}{c}\text { Chicos } \\
n(\%)\end{array}$ & $\begin{array}{c}\text { Chicas } \\
n(\%)\end{array}$ & $\chi^{2}$ & V \\
\hline \multirow[t]{2}{*}{ Víctima autoinformada } & No & $214(69.0 \%)$ & $209(62.8 \%)$ & 2.80 & .07 \\
\hline & Sí & $96(31.0 \%)$ & $124(37.2 \%)$ & & \\
\hline \multirow[t]{2}{*}{ Víctima heteroinformada } & No & $278(85.5 \%)$ & $303(90.2 \%)$ & 3.34 & .07 \\
\hline & Sí & $47(14.5 \%)$ & $33(9.8 \%)$ & & \\
\hline \multirow[t]{2}{*}{ Bully ejercido autoinformado } & No & $193(62.1 \%)$ & $245(73.4 \%)$ & $9.43 * *$ & .12 \\
\hline & Sí & $118(37.9 \%)$ & $89(26.6 \%)$ & & \\
\hline \multirow[t]{2}{*}{ Bully heteroinformado } & No & $262(80.6 \%)$ & $307(91.4 \%)$ & $15.94 * * *$ & .16 \\
\hline & Sí & $63(19.4 \%)$ & $29(8.6 \%)$ & & \\
\hline
\end{tabular}

$* * p<.01 ; * * * p<.001$.

Diferencias en las variables de ajuste psicológico en función del perfil autoinformado

Tras los análisis preliminares, se compararon a través de ANOVA y contrastes post hoc las diferencias en los niveles en las variables de ajuste psicológico en función del perfil en las respuestas autoinformadas. Los resultados se muestran en la Tabla 2. En lo que se refiere a la autoestima, el grupo de control y el grupo de los agresores obtuvieron puntuaciones significativamente mayores que las víctimas y que los agresores-víctimas. No hubo diferencias entre el grupo de control y los agresores en la variable autoestima.

En la puntuación total de autocompasión, el grupo de control obtuvo puntuaciones significativamente mayores que las víctimas y que los agresores-víctimas, pero no diferentes a los agresores, con unos tamaños del efecto próximos a medios. En self-warmth se encontraron diferencias significativas entre el grupo de control y los agresores. En la subescala self-coldness, el grupo de control y el grupo de los agresores obtuvieron unas puntuaciones significativamente menores que los grupos de víctimas y de agresores-víctimas. El grupo de control y el grupo de los agresores puntuaron significativamente más bajo que el grupo de las víctimas en afecto negativo. Sin embargo, no hubo diferencias en el afecto positivo.

En resumen, el grupo de los agresores y el grupo de control mostraron unas puntuaciones similares en las variables de ajuste psicológico, exceptuando la variable self-warmth; y las puntuaciones de estos dos grupos fueron significativamente más favorables desde el punto de vista del ajuste psicológico que las de los grupos de agresores-víctimas y las víctimas. No se encontraron diferencias significativas entre estos dos últimos grupos en las variables analizadas, salvo en self-warmth

\section{Diferencias en las variables de ajuste psicológico tras la inclusión de medidas heteroinformadas}

Tras examinar las diferencias en función del perfil autoinformado, se analizó el ajuste psicológico de los participantes, una vez incluidas medidas heteroinformadas. Los resultados en las variables de ajuste psicológico en función de la victimización auto- y heteroinformada se muestran en la Tabla 3.

En la variable autoestima el grupo de control obtuvo puntuaciones significativamente mayores que los dos grupos que informaban sufrir acoso. También, las víctimas auto- $y$ heteroinformadas obtuvieron puntuaciones significativamente más bajas que aquellos que eran señalados por sus compañeros como posibles víctimas, pero no se reconocían en ese rol. En las variables autocompasión total y self-coldness, se encontraron diferencias significativas entre los grupos de modo similar a las encontrados en la autoestima, solo que con una diferencia adicional en self-coldness, en la que las víctimas heteroinformadas obtuvieron una puntuación significativamente mayor que las víctimas autoinformadas. No hubo diferencias significativas entre los grupos en afecto positivo, pero sí en afecto negativo. Los dos grupos que no declararon sufrir acoso puntuaron significativamente más bajo que los dos que declaraban sufrirlo. Los resultados en las variables de ajuste psicológico en función de la participación o no como agresor se muestran en la Tabla 4. 
Tabla 2. Diferencias en las variables de ajuste psicológico dependiendo del perfil autoinformado

\begin{tabular}{|c|c|c|c|c|c|c|c|}
\hline & \multirow{2}{*}{$\begin{array}{l}\text { Control } \\
M(D T) \\
n=314\end{array}$} & \multirow{2}{*}{$\begin{array}{l}\text { Víctimas } \\
M(D T) \\
n=124\end{array}$} & \multirow{2}{*}{$\begin{array}{c}\text { Agreso- } \\
\text { res-víctimas } \\
M(D T) \\
n=97\end{array}$} & \multirow{2}{*}{$\begin{array}{c}\text { Agresores } \\
M(D T) \\
n=109\end{array}$} & \multicolumn{2}{|c|}{ ANOVA } & \multirow[b]{2}{*}{$d$} \\
\hline & & & & & $F$ & Tests Post Hoc ${ }^{\mathrm{a}}$ & \\
\hline \multirow[t]{4}{*}{ Autoestima } & $3.19(.47)$ & $2.98(.46)$ & $3.04(.43)$ & $3.20(.38)$ & $8.41^{* * *}$ & $\mathrm{C}>\mathrm{V}^{* * *}$ & .45 \\
\hline & & & & & & $\mathrm{C}>\mathrm{AV}^{*}$ & .33 \\
\hline & & & & & & $\mathrm{V}<\mathrm{A}^{* * *}$ & .52 \\
\hline & & & & & & $\mathrm{AV}<\mathrm{A}^{*}$ & .13 \\
\hline \multirow[t]{2}{*}{ Autocompasión total } & $3.27(.61)$ & $3.01(.65)$ & $2.99(.49)$ & $3.11(.53)$ & $9.63^{* * *}$ & $\mathrm{C}>\mathrm{V}^{* * *}$ & .41 \\
\hline & & & & & & $\mathrm{C}>\mathrm{AV}^{* * *}$ & .51 \\
\hline \multirow[t]{4}{*}{ Self-coldness } & $2.65(.83)$ & $3.05(.82)$ & $3.07(.70)$ & $2.78(.76)$ & $11.28^{* * *}$ & $\mathrm{C}<\mathrm{V}^{* * *}$ & .48 \\
\hline & & & & & & $\mathrm{C}<\mathrm{AV}^{* * *}$ & .55 \\
\hline & & & & & & $\mathrm{V}>\mathrm{A}^{*}$ & .34 \\
\hline & & & & & & $\mathrm{AV}>\mathrm{A}^{*}$ & .39 \\
\hline Self-warmth & $3.22(.69)$ & $3.07(.74)$ & $3.06(.60)$ & $3.01(.66)$ & $3.65^{*}$ & $\mathrm{C}>\mathrm{A}^{*}$ & .31 \\
\hline Afecto positivo & $2.38(.71)$ & $2.41(.71)$ & $2.46(.59)$ & $2.39(.62)$ & & & \\
\hline \multirow[t]{3}{*}{ Afecto negativo } & $.98(0.69)$ & $1.34(.77)$ & $1.28(.72)$ & $1.09(.62)$ & $9.79 * * *$ & $\mathrm{C}<\mathrm{V}^{* * *}$ & .49 \\
\hline & & & & & & $\mathrm{C}<\mathrm{AV}^{* *}$ & .42 \\
\hline & & & & & & $\mathrm{V}>\mathrm{A}^{* *}$ & .36 \\
\hline
\end{tabular}

Nota $. \mathrm{A}=$ agresores; $\mathrm{AV}=$ agresores-víctimas; $\mathrm{C}=$ control; $\mathrm{V}=$ víctimas. ${ }^{\mathrm{a} T e s t}$ post hoc realizados con la corrección de Bonferroni. ${ }^{*} p<$ $.05 ; * * p<.01 ; * * *<.001$.

En lo que respecta a la autoestima, no se encontraron diferencias significativas entre los grupos. En la puntuación total de autocompasión, no se encontraron diferencias significativas entre los agresores heteroinformados y el grupo de control. Sí se encontraron diferencias entre estos dos grupos y el grupo de agresores auto y heteroinformados. Además, los alumnos del grupo de control mostraron puntuaciones significativamente mayores en la subescala self-warmth que los alumnos que declaraban ejercer el acoso y que eran señalados por sus compañeros como agresores.

\section{Discusión}

Los resultados encontrados en prevalencia del bullying se encuentran en el rango de lo informado en otros estudios (Craig et al., 2009; Díaz-Aguado Jalón et al., 2013). Sin embargo, se detectaron más casos a través de medidas heteroinformadas que a través de medidas autoinformadas. Esto no quiere decir que el porcentaje dado a través de medidas heteroinformadas sea correcto y el otro no. Sin embargo, sí que hay casos no recogidos en las medidas autoinformadas que parecen estar su- friendo bullying y viceversa. Por lo tanto, parece necesario el uso de ambos tipos de medida para la obtención de datos de prevalencia más acertados y detectar posibles situaciones de acoso (Babarro, 2014). Si bien existe cierto consenso en el uso de la escala de Olweus para medir el bullying autoinformado, no existe tal consenso respecto a las escalas heteroinformadas y sería recomendable alcanzarlo, tanto para estudiar la prevalencia, como para la detección de los casos de riesgo en los centros educativos.

En lo que se refiere a las diferencias en el acoso en función del género, los chicos mostraron una tendencia significativamente mayor a ser agresores tanto en medidas autoinformadas como heteroinformadas, lo que es acorde a la mayoría de estudios realizados hasta la fecha (Díaz-Aguado Jalón et al., 2013; Smith et al., 2019). No hubo diferencias significativas en la victimización dependiendo del género, ni en las medidas autoinformadas ni en las heteroinformadas. Sí se encontró que en las medidas autoinformadas las chicas informaban de sufrir más acoso, y sin embargo en las medidas heteroinformadas eran los chicos los que eran señalados en mayor medida por sus compañeros como posibles víctimas de aco- 
Tabla 3. Medias, desviaciones típicas y diferencias entre los distintos grupos dependiendo de que el bullying sea autoinformado o heteroinformado

\begin{tabular}{|c|c|c|c|c|c|c|c|}
\hline & \multirow{2}{*}{$\begin{array}{l}\text { Grupo } 1 \\
M(D T) \\
n=43\end{array}$} & \multirow{2}{*}{$\begin{array}{l}\text { Grupo } 2 \\
M(D T) \\
n=178\end{array}$} & \multirow{2}{*}{$\begin{array}{l}\text { Grupo } 3 \\
M(D T) \\
n=35\end{array}$} & \multirow{2}{*}{$\begin{array}{l}\text { Grupo } 4 \\
M(D T) \\
n=388\end{array}$} & \multicolumn{2}{|c|}{ ANOVA } & \multirow{2}{*}{$d$} \\
\hline & & & & & $F$ & Tests Post Hoc ${ }^{a}$ & \\
\hline \multirow[t]{3}{*}{ Autoestima } & $2.91(.45)$ & $3.17(.47)$ & $3.03(.45)$ & $3.19(.45)$ & $8.96 * * *$ & $1<2 *$ & .56 \\
\hline & & & & & & $1<4 * * *$ & .62 \\
\hline & & & & & & $3<4 * * *$ & .35 \\
\hline \multirow[t]{3}{*}{ Autocompasión total } & $2.92(.56)$ & $3.27(.57)$ & $3.02(.59)$ & $3.23(.60)$ & $7.98 * * *$ & $1<2 *$ & .62 \\
\hline & & & & & & $1<4 * *$ & .25 \\
\hline & & & & & & $3<4 * * *$ & .35 \\
\hline \multirow[t]{4}{*}{ Self-coldness } & $3.24(.75)$ & $2.53(.85)$ & $3.01(.77)$ & $2.70(.81)$ & $12,12 * * *$ & $1>2 * * *$ & .89 \\
\hline & & & & & & $1>4 * * *$ & .69 \\
\hline & & & & & & $2>3 * *$ & .59 \\
\hline & & & & & & $3>4 * * *$ & .39 \\
\hline Self-warmth & $3.09(.65)$ & $3.10(.68)$ & $3.06(.69)$ & $3.17(.69)$ & 1.28 & & \\
\hline Afecto positivo & $2.33(.76)$ & $2.34(.72)$ & $2.46(.63)$ & $2.39(.68)$ & 0.68 & & \\
\hline \multirow[t]{3}{*}{ Afecto negativo } & $1.45(.72)$ & $1.01(.67)$ & $1.28(.75)$ & $1.01(.68)$ & $9.65 * * *$ & $1>2 *$ & .23 \\
\hline & & & & & & $1>4 * * *$ & .63 \\
\hline & & & & & & $3>4 * * *$ & .38 \\
\hline
\end{tabular}

Nota. Grupo 1 = víctimas auto- y heteroinformadas; Grupo 2 = víctimas solo heteroinformadas; Grupo $3=$ víctimas solo autoinformadas; Grupo 4 = alumnos que no autoinforman ni son heteroinformados como víctimas. ${ }^{*} p<.05 ; * * p<.01 ; * * *<.001$. ${ }^{\text {a Test }}$ post hoc realizados con la corrección de Bonferroni.

Tabla 4. Diferencias en las variables de ajuste psicológico dependiendo de que el bullying ejercido sea autoinformado, heteroinformado o ambos

\begin{tabular}{|c|c|c|c|c|c|c|c|}
\hline & \multirow{2}{*}{$\begin{array}{l}\text { Grupo A } \\
M(D T) \\
n=41\end{array}$} & \multirow{2}{*}{$\begin{array}{l}\text { Grupo B } \\
M(D T) \\
n=48\end{array}$} & \multirow{2}{*}{$\begin{array}{l}\text { Grupo C } \\
M(D T) \\
n=166\end{array}$} & \multirow{2}{*}{$\begin{array}{l}\text { Grupo D } \\
M(D T) \\
n=391\end{array}$} & \multicolumn{2}{|c|}{ ANOVA } & \multirow{2}{*}{$d$} \\
\hline & & & & & $F$ & Tests Post Hoc ${ }^{\mathrm{a}}$ & \\
\hline Autoestima & $3.09(.37)$ & $3.23(.42)$ & $3.13(.42)$ & $3.12(.49)$ & & & \\
\hline \multirow[t]{2}{*}{ Autocompasión total } & $2.96(.50)$ & $3.24(.56)$ & $3.08(.51)$ & $3.19(.64)$ & $3.32 *$ & $\mathrm{~A}<\mathrm{B}^{*}$ & .53 \\
\hline & & & & & & $\mathrm{A}<\mathrm{D}^{*}$ & .40 \\
\hline Self-coldness & $2.97(.72)$ & $2.64(.81)$ & $2.90(.75)$ & $2.78(.84)$ & & & \\
\hline Self-warmth & $2.89(.64)$ & $3.16(.59)$ & $3.06(.63)$ & $3.18(.72)$ & $2.87^{*}$ & $\mathrm{~A}<\mathrm{D}^{*}$ & .42 \\
\hline Afecto positivo & $2.43(.62)$ & $2.52(.72)$ & $2.41(.60)$ & $2.38(.71)$ & 0.72 & & \\
\hline Afecto negativo & $1.28(.67)$ & $.95(.75)$ & $1.16(.67)$ & $1.10(.73)$ & 1.79 & & \\
\hline
\end{tabular}

Nota. Grupo A = agresores auto- y heteroinformados; Grupo B = agresores solo heteroinformados; Grupo C = agresores solo autoinformados; Grupo D = alumnos que no autoinforman ni son heteroinformados como agresores. ${ }^{*} p<.05,{ }^{*} p<.01,{ }^{* * *} p<.001$. ${ }^{\mathrm{a}}$ Test Post Hoc realizados con la corrección de Bonferroni. 
so. Una posible explicación a estos datos es que las chicas sean más conscientes del acoso y tengan actitudes más claras contrarias al mismo, por lo que lo reconocen con más facilidad (Trach et al, 2010). Otra posible explicación es que para los chicos sea más difícil reconocerse en el rol de víctima. Un último argumento posible es que el tipo de violencia que sufren las chicas sea más sutil (por ej., violencia social) (Trach et al, 2010), y solo lo detecte la propia víctima, pero no el resto de compañeros. Investigaciones que empleen otras medidas o evalúen de manera cualitativa los casos, podrán ayudar a encontrar la explicación más acertada. En cualquier caso, en la literatura existente, las diferencias de género en victimización se han basado ampliamente en medidas autoinformadas y, hasta el momento, han encontrado resultados contradictorios. En este estudio, el empleo conjunto de ambas medidas ayuda a encontrar nuevos matices que pueden aclarar la cuestión.

En las medidas autoinformadas, los resultados muestran que el ajuste psicológico medido a través de la autoestima y el afecto positivo y negativo es similar entre los no implicados y los agresores; en ambos casos éste es mejor que el de las víctimas y que el de los agresores-víctimas. No ocurrió así en el caso de la escala self-warmth que recoge los aspectos más positivos de la autocompasión. Estos resultados, por tanto, van en la línea de otras publicaciones que no han detectado mayores problemas de ajuste psicológico en los agresores que en los alumnos no implicados directamente (Cerezo, 2009; Estévez et al., 2009; Rigby y Slee, 1991). Por otro lado, no se encontraron diferencias significativas entre las víctimas y los agresores-víctimas en ninguna de las medidas examinadas, lo que es acorde a otros estudios con poblaciones similares (Estévez et al., 2009).

Tras la inclusión de las medidas heteroinformadas, los resultados muestran, en el caso de las víctimas, que el reconocerse en el rol de víctima es lo que guarda una mayor asociación con el ajuste psicológico, de tal modo que aquellos que son señalados por sus compañeros como posibles víctimas, pero no se reconocen en ese rol, tienen un ajuste psicológico similar a los que no son víctimas. Sin embargo, los que se reconocen en ese rol, aunque sus compañeros no los señalen como víctimas, tienen un peor ajuste psicológico. Aquellos que se reconocieron como víctima y además fueron señalados por sus compañeros como víctimas, tuvieron las peores puntuaciones en las variables de ajuste psicológico, probablemente porque esas situaciones de acoso eran especialmente graves. Estos resultados apoyan la importancia de la subjetividad en el proceso de victimización y está relacionada con los estudios que han encontrado una relación bidireccional entre cierta sintomatología psicopa- tológica y victimización (Kochel et al., 2012; Sweeting et al., 2006).

En el caso de los agresores se encontraron menos diferencias en el ajuste psicológico que en el caso de las víctimas. Aquellos que se reconocieron en el rol de agresores y que fueron señalados por sus compañeros como tales mostraron un peor ajuste psicológico que los del grupo de control y que aquellos que no se reconocían en ese rol. Los agresores pueden no reconocer su rol en el acoso debido a la desconexión moral, porque contestan en función de la deseabilidad social o simplemente porque desconfían de la anonimidad del test (Solberg y Olweus, 2003). Además, los agresores, especialmente en secundaria, tienden a realizar las agresiones respaldados por el grupo y les suele servir para ganar apoyo y estatus, y, además, tienden a mostrar poco conocimiento del daño que hacen en el otro y a excusar sus acciones o a culpabilizar a la víctima, por tanto, estos resultados no son extraños.

Otro objetivo de la presente investigación fue comparar la variable autoestima frente a la autocompasión en el contexto de la adolescencia y la dinámica del bullying. Uno de los motivos de que el estudio de la autocompasión haya proliferado tanto en los últimos años es un descontento con algunos matices del concepto de autoestima y con sus implicaciones; por ejemplo, algunas investigaciones han encontrado que los agresores del bullying tienen una autoestima superior o igual a los no implicados (Cerezo, 2009), que llevaban a cuestionar lo saludable de tener una «alta» autoestima (Baumeister et al., 2003; Leary y Baumeister, 2000; Neff, 2009). Los resultados aquí presentados indican que el comportamiento de ambas variables es diferente. En las medidas autoinformadas, agresores y no implicados puntuaron igual en autoestima; sin embargo, en la variable self-warmth los no implicados obtuvieron mayores puntuaciones, lo que nos lleva a pensar que los no implicados tienen una forma más saludable de relacionarse consigo mismos. Así mismo, las víctimas que son señaladas por sus compañeros, pero no se reconocen en ese rol, tienden a tener una mayor autocompasión, derivada principalmente, no tanto de un buen trato hacia sí mismas (self-warmth), sino de no tratarse de un modo cruel (self-coldness), lo que da respaldo a considerar la autocompasión como un importante recurso para amortiguar las consecuencias del acoso y la victimización (Bluth y Blanton, 2015; Játiva y Cerezo, 2014). Por último, aquellos alumnos que son señalados por sus compañeros como agresores, pero que no se reconocen en ese rol, puntúan más alto en autocompasión que los agresores que sí se reconocen en ese rol. Esto nos lleva a pensar que, a pesar de que en teoría la autocompasión es dife- 
rente de la indulgencia para con uno mismo (Germer y Neff, 2013), es muy posible que la diferencia sea sutil para algunos alumnos de esta edad, o que la adaptación al español no recoja con precisión este matiz.

Es necesario señalar que el presente estudio cuenta con algunas limitaciones. En primer lugar, especialmente en las medidas autoinformadas y por la naturaleza correlacional del estudio, no podemos concluir si tener un peor estado psicológico lleva a una mayor sensibilidad a la hora de sentirse víctima de acoso o si, por el contrario, sufrir acoso es lo que lleva a ese peor estado psicológico. Aunque parece que puede existir cierta vulnerabilidad psicopatológica previa en algunas víctimas, los estudios longitudinales apuntan a que el acoso, con vulnerabilidad previa o no, tiene graves consecuencias sobre la salud psicológica (Moore et al., 2017). En segundo lugar, aunque las medidas heteroinformadas han mostrado una fiabilidad alta, cabe la duda de si están midiendo lo mismo que las medidas autoinformadas, especialmente en el caso de los agresores, y de ahí, que haya casos que no se correspondan. En cualquier caso, parece que el uso conjunto de ambos tipos de medidas es de gran ayuda tanto para dilucidar temas controvertidos en la investigación, como para detectar situaciones de acoso que se escapan a las medidas autoinformadas y viceversa. En tercer lugar, no se ha incluido el perfil de agresor-víctima heteroinformado, con lo que no podemos descartar que algunas de las víctimas sean también agresores o al revés. Esto supone un reto a nivel metodológico para futuras investigaciones; ya que los alumnos pueden contestar con un efecto halo y les es difícil categorizar a una misma persona en los dos perfiles. Además, el tamaño de la muestra ha de ser mayor para permitir la creación de subgrupos de tamaño comparable. Por último, es posible que los resultados comparativos encontrados entre los agresores auto- y heteroinformados puedan deberse a que las pruebas detectan perfiles diferentes dentro de los agresores, un perfil más caracterizado por una mala regulación emocional que desemboca en agresiones reactivas hacia otros miembros de la clase, o un perfil más proactivo que ejerce la violencia de un modo instrumental para ganar estatus y poder en la clase (Avilés et al., 2011; Salmivalli y Nieminen, 2002). Medidas auto- y heteroinformadas que ayuden a distinguir estos perfiles podrían suponer una mejora a la hora de aclarar cómo es el ajuste psicológico de los agresores.

Contando con las mencionadas limitaciones, el presente estudio aporta conclusiones relevantes respecto al estudio de la asociación entre bullying, autoestima, afecto y autocompasión. En primer lugar, la presente investigación ofrece respaldo al uso conjunto de medidas autoinformadas y heteroinformadas para aclarar algunas de las cuestiones pendientes de resolver en la literatura sobre el bullying y sus consecuencias. En segundo lugar, se resalta la importancia de la subjetividad de agresores y víctimas en las repercusiones psicológicas del acoso. En tercer lugar, aclara el comportamiento diferencial de las variables autoestima y autocompasión en relación al acoso. Estas conclusiones son relevantes para la prevención e intervención clínica y educativa con alumnos víctimas o agresores de bullying.

\section{Conflicto de intereses}

Los autores declaran que no existen conflictos de intereses.

\section{Referencias}

Avilés, J. M., Irurtia, M. J., García-Lopez, L. J. y Caballo, V. E. (2011). El maltrato entre iguales: «Bullying». Psicología Conductual, 19(1), 57-90. https://www.behavioralpsycho. com/wp-content/uploads/2019/08/04.Avilés_19-1oa.pdf

Babarro, J. M. (2014). Assessment and detection of peer-bullying through analysis of the group context. Psicothema, 26(3), 357-363. http://www.psicothema.com/pdf/4200.pdf

Bang, Y. R. \& Park, J. H. (2017). Psychiatric disorders and suicide attempts among adolescents victimized by school bullying. Australasian Psychiatry, 25(4), 376-380. http://dx.doi. org/10.1177/1039856217715987

Baumeister, R. F., Campbell, J. D., Krueger, J. I., \& Vohs, K. D. (2003). Does high self-esteem cause better performance, interpersonal success, happiness, or healthier lifestyles? Psychological Science in the Public Interest, 4(1), 1-44. http:// dx.doi.org/10.1111/1529-1006.01431

Björkqvist, K., Österman, K., \& Kaukiainen, A. (1992). The development of direct and indirect aggressive strategies in males and females. En K. Björkqvist y P. Niemela (Eds.), Of mice and women: Aspects of female aggression (pp. 51-64). Academic Press. http://dx.doi.org/10.1016/B978-0-12102590-8.50010-6

Bluth, K. \& Blanton, P. W. (2015). The influence of selfcompassion on emotional well-being among early and older adolescent males and females. The Journal of Positive Psychology, 10(3), 219-230. http://dx.doi.org/10.1080/17439 760.2014 .936967

Bluth, K. \& Neff, K. D. (2018). New frontiers in understanding the benefits of self-compassion. Self and Identity, 17(6), 605608. http://dx.doi.org/10.1080/15298868.2018.1508494

Brunstein Klomek, A., Barzilay, S., Apter, A., Carli, V., Hoven, C. W., Sarchiapone, M., Hadlaczky, G., Balazs, J., Kereszteny, A., Brunner, R., Kaess, M., Bobes, J., Saiz P.A, Cosman, D., Haring C., Banzer, R., McMahon, E., Keeley, H., Kahn, J.P., Postuvan, V., Podlogar, T., Sisask M., Varnik, A., \& Wasserman, D. (2019). Bi-directional longitudinal associations between different types of bullying victimization, suicide ideation/ attempts, and depression among a large sample of European adolescents. Journal of Child Psychology and Psychiatry, 60(2), 209-215. http://dx.doi.org/10.1111/jcpp.12951 
Castilho, P., Carvalho, S. A., Marques, S., \& Pinto-Gouveia, J. (2017). Self-compassion and emotional intelligence in adolescence: A multigroup mediational study of the impact of shame memories on depressive symptoms. Journal of Child and Family Studies, 26(3), 759-768. http://dx.doi.org/10.1007/ s10826-016-0613-4

Cerezo, F. (2009). Bullying: Análisis de la situación en las aulas españolas. International Journal of Psychology and Psychological Therapy, 9(3), 383-394. https://www.ijpsy.com/ volumen9/num3/244/bullying-anlisis-de-la-situacin-en-lasES.pdf

Craig, W., Harel-Fisch, Y., Fogel-Grinvald, H., Dostaler, S., Hetland, J., Simons-Morton, B., Molcho, M., de Mato, M. G., Overpeck, M., Due, P., Pickett, W., HBSC Violence \& Injuries Prevention Focus Group, \& HBSC Bullying Writing Group. (2009). A cross-national profile of bullying and victimization among adolescents in 40 countries. International Journal of Public Health, 54(2), 216-224. http://dx.doi.org/10.1007/ s00038-009-5413-9

Crick, N. R. \& Grotpeter, J. K. (1995). Relational aggression, gender, and social-psychological adjustment. Child Development, 66(3), 710-722. http://dx.doi.org/10.2307/1131945

Díaz-Aguado Jalón, M. J., Martínez Arias, R. y Martín Babarro, J. (2013). El acoso entre adolescentes en España. Prevalencia, papeles adoptados por todo el grupo y características a las que atribuyen la victimización. Revista de Educación, 362, 348379. http://www.revistaeducacion.educacion.es/doi/362_164. pdf

Echeburúa, E. y Arbona, C. B. (1995). Evaluación y tratamiento de la fobia social. Martínez Roca.

Edwards, M., Adams, E. M., Waldo, M., Hadfield, O. D., \& Biegel, G. M. (2014). Effects of a mindfulness group on Latino adolescent students: Examining levels of perceived stress, mindfulness, self-compassion, and psychological symptoms. The Journal for Specialists in Group Work, 39(2), 145-163. http://dx.doi.org/10.1080/01933922.2014.891683

Estévez, E., Murgui, S., \& Musitu, G. (2009). Psychological adjustment in bullies and victims of school violence. European Journal of Psychology of Education, 24(4), 473. http://dx.doi. org/10.1007/BF03178762

Galla, B. M. (2016). Within-person changes in mindfulness and self-compassion predict enhanced emotional well-being in healthy, but stressed adolescents. Journal of Adolescence, 49, 204-217. http://dx.doi.org/10.1016/j.adolescence.2016.03.016

Garcia-Campayo, J., Navarro-Gil, M., Andrés, E., Montero-Marin, J., López-Artal, L., \& Demarzo, M. M. P. (2014). Validation of the Spanish versions of the long (26 items) and short (12 items) forms of the Self-Compassion Scale (SCS). Health and Quality of Life Outcomes, 12, 4. https://doi.org/10.1186/14777525-12-4

Germer, C. K., \& Neff, K. D. (2013). Self-compassion in clinical practice. Journal of Clinical Psychology, 69(8), 856-867. http://dx.doi.org/10.1002/jclp.22021

Gilbert, P., McEwan, K., Matos, M., \& Rivis, A. (2011). Fears of compassion: Development of three self-report measures. Psychology and Psychotherapy: Theory, Research and Practice, 84(3), 239-255. http://dx.doi.org/10.1348/147608310X526511

Hinduja, S. \& Patchin, J. W. (2019). Connecting adolescent suicide to the severity of bullying and cyberbullying. Journal of School Violence, 18(3), 333-346. http://dx.doi.org/10.1080/ 15388220.2018 .1492417

Houbre, B., Tarquinio, C., Thuillier, I., \& Hergott, E. (2006). Bullying among students and its consequences on health. European Journal of Psychology of Education, 21(2), 183208. http://dx.doi.org/10.1007/BF03173576

Játiva, R. \& Cerezo, M. A. (2014). The mediating role of selfcompassion in the relationship between victimization and psychological maladjustment in a sample of adolescents. Child Abuse \& Neglect, 38(7), 1180-1190. http://dx.doi. org/10.1016/j.chiabu.2014.04.005

Khatri, P., Kupersmidt, J. B., \& Patterson, C. (2000). Aggression and peer victimization as predictors of self-reported behavioral and emotional adjustment. Aggressive Behavior: Official Journal of the International Society for Research on Aggression, 26(5), 345-358. http://dx.doi.org/10.1002/10982337(2000)26:5\%3C345::AID-AB1\%3E3.0.CO;2-L

Kochel, K. P., Ladd, G. W., \& Rudolph, K. D. (2012). Longitudinal associations among youth depressive symptoms, peer victimization, and low peer acceptance: An interpersonal process perspective. Child Development, 83(2), 637-650. http://dx.doi.org/10.1111/j.1467-8624.2011.01722.x

Kowalski, R. M. \& Limber, S. P. (2013). Psychological, physical, and academic correlates of cyberbullying and traditional bullying. Journal of Adolescent Health, 53(1), S13-S20. http:// dx.doi.org/10.1016/j.jadohealth.2012.09.018

Leary, M. R. \& Baumeister, R. F. (2000). The nature and function of self-esteem: Sociometer theory. Advances in experimental social psychology (pp. 1-62). Elsevier. http://dx.doi. org/10.1016/S0065-2601(00)80003-9

Lie, S. Ø, Rø, Ø, \& Bang, L. (2019). Is bullying and teasing associated with eating disorders? A systematic review and meta-analysis. International Journal of Eating Disorders, 52(5), 497-514. http://dx.doi.org/10.1002/eat.23035

López, A., Sanderman, R., Smink, A., Zhang, Y., Van Sonderen, E., Ranchor, A., \& Schroevers, M. J. (2015). A reconsideration of the self-compassion scale's total score: Self-compassion versus self-criticism. PloS One, 10(7), e0132940. http://dx. doi.org/10.1371/journal.pone.0132940

MacDonald, J. M., Piquero, A. R., Valois, R. F., \& Zullig, K. J. (2005). The relationship between life satisfaction, risk-taking behaviors, and youth violence. Journal of Interpersonal Violence, 20(11), 1495-1518. http://dx.doi.org/10.1177/0886260505278718

Magaz, A., Chorot, P., Sandín, B., Santed, M.A. y Valiente, R.M. (2011). Estilos de apego y acoso entre iguales (bullying) en adolescentes. Revista de Psicopatología y Psicología Clínica, 16(3), 207-221. https://doi.org/10.5944/rppc.vol.16. num.3.2011.10362

Magaz, A., Chorot, P., Santed, M.A., Valiente, R.M. y Sandín, B. (2016). Evaluación del bullying como victimización: Estructura, fiabilidad y validez del Cuestionario de Acoso entre Iguales (CAI). Revista de Psicopatología y Psicología Clínica, 21(2), 77-95. https://doi.org/10.5944/rppc.vol.21. num.2.2016.16990

Moore, S. E., Norman, R. E., Sly, P. D., Whitehouse, A. J., Zubrick, S. R., \& Scott, J. (2014). Adolescent peer aggression and its association with mental health and substance use in an Australian cohort. Journal of Adolescence, 37(1), 11-21. http://dx.doi.org/10.1016/j.adolescence.2013.10.006 
Moore, S. E., Norman, R. E., Suetani, S., Thomas, H. J., Sly, P. D., \& Scott, J. G. (2017). Consequences of bullying victimization in childhood and adolescence: A systematic review and metaanalysis. World Journal of Psychiatry, 7(1), 60-76. http://dx. doi.org/10.5498/wjp.v7.i1.60

Natvig, G. K., Albrektsen, G., \& Qvarnstrøm, U. (2001). Schoolrelated stress experience as a risk factor for bullying behavior. Journal of Youth and Adolescence, 30(5), 561-575. http://dx. doi.org/10.1023/A:1010448604838

Neff, K. D. (2003). The development and validation of a scale to measure self-compassion. Self and Identity, 2(3), 223-250. http://dx.doi.org/10.1080/15298860309027

Neff, K. D. (2009). The role of self-compassion in development: A healthier way to relate to oneself. Human Development, 52(4), 211-214. http://dx.doi.org/10.1159/000215071

Obando, D., Trujillo, Á. y Prada, M. (2018). Conducta autolesiva no suicida en adolescentes y su relación con factores personales y contextuales. Revista de Psicopatología y Psicología Clínica, 23(3), 189-200. http://dx.doi.org/10.5944/ rppc.vol.23.num.3.2018.21278

Olweus, D. (1994). Long-term outcomes for victims and an effective school-based intervention program. En L.R. Huesman (ED.), Aggressive Behavior: Current Perspectives, (pp. 97-130). Plenum Press. https://doi.org/10.1007/978-14757-9116-7_5

Olweus, D. (1996). The Revised Olweus Bully/Victim Questionnaire. Bergen, Norway: Research Center for Health Promotion (HEMIL center), Universidad de Bergen.

Pedreira-Massa, J. L. (2019). Conductas suicidas en la adolescencia: Una guía práctica para la intervención y la prevención. Revista de Psicopatología y Psicología Clínica, 24(3), 217-237. http:// dx.doi.org/10.5944/rppc. 26280

Pellegrini, A. D., Bartini, M., \& Brooks, F. (1999). School bullies, victims, and aggressive victims: Factors relating to group affiliation and victimization in early adolescence. Journal of Educational Psychology, 91(2), 216-224. http://dx.doi. org/10.1037/0022-0663.91.2.216

Rigby, K., \& Slee, P. T. (1991). Bullying among Australian school children: Reported behavior and attitudes toward victims. The Journal of Social Psychology, 131(5), 615-627. http://dx.doi. org/10.1080/00224545.1991.9924646

Rosenberg, M. (1965). Rosenberg self-esteem scale (RSE). Acceptance and Commitment Therapy. Measures Package, 61(52), 18.

Salmivalli, C., Kaukiainen, A., Kaistaniemi, L., \& Lagerspetz, K. M. (1999). Self-evaluated self-esteem, peer-evaluated selfesteem, and defensive egotism as predictors of adolescents' participation in bullying situations. Personality and Social Psychology Bulletin, 25(10), 1268-1278. http://dx.doi. org/10.1177/0146167299258008

Salmivalli, C. \& Nieminen, E. (2002). Proactive and reactive aggression among school bullies, victims, and bully-victims. Aggressive Behavior: Official Journal of the International
Society for Research on Aggression, 28(1), 30-44. http://dx. doi.org/10.1002/ab.90004

Sandín, B. (2003). Escalas PANAS de afecto positivo y negativo para niños y adolescentes (PANASN). Revista de Psicopatología y Psicología Clínica, 8(2), 173-182. http:// dx.doi.org/10.5944/rppc.vol.8.num.2.2003.3953

Schwartz, D., Gorman, A. H., Nakamoto, J., \& Toblin, R. L. (2005). Victimization in the peer group and children's academic functioning. Journal of Educational Psychology, 97(3), 425-435. http://dx.doi.org/10.1037/0022-0663.97.3.425

Smith, P. K., López-Castro, L., Robinson, S., \& Görzig, A. (2019). Consistency of gender differences in bullying in cross-cultural surveys. Aggression and Violent Behavior, 45, 33-40. http:// dx.doi.org/10.1016/j.avb.2018.04.006

Solberg, M. E. \& Olweus, D. (2003). Prevalence estimation of school bullying with the Olweus Bully/Victim Questionnaire. Aggressive Behavior: Official Journal of the International Society for Research on Aggression, 29(3), 239-268. http:// dx.doi.org/10.1002/ab.10047

Swearer, S. M., Song, S. Y., Cary, P. T., Eagle, J. W., \& Mickelson, W. T. (2001). Psychosocial correlates in bullying and victimization: The relationship between depression, anxiety, and bully/victim status. Journal of Emotional Abuse, 2(2-3), 95-121. http://dx.doi.org/10.1300/J135v02n02_07

Sweeting, H., Young, R., West, P., \& Der, G. (2006). Peer victimization and depression in early-mid adolescence: A longitudinal study. British Journal of Educational Psychology, 76(3), 577-594. http://dx.doi.org/10.1348/000709905X49890

Trach, J., Hymel, S., Waterhouse, T., \& Neale, K. (2010). Bystander responses to school bullying: A cross-sectional investigation of grade and sex differences. Canadian Journal of School Psychology, 25(1), 114-130. http://dx.doi. org/10.1177/0829573509357553

Vigna, A. J., Poehlmann-Tynan, J., \& Koenig, B. W. (2018). Does self-compassion facilitate resilience to stigma? A schoolbased study of sexual and gender minority youth. Mindfulness, 9(3), 914-924. http://dx.doi.org/10.1007/s12671-017-0831-x

Vivolo-Kantor, A. M., Martell, B. N., Holland, K. M., \& Westby, R. (2014). A systematic review and content analysis of bullying and cyber-bullying measurement strategies. Aggression and Violent Behavior, 19(4), 423-434. http://dx. doi.org/10.1016/j.avb.2014.06.008

Xavier, A., Gouveia, J. P., \& Cunha, M. (2016). Non-suicidal selfinjury in adolescence: The role of shame, self-criticism and fear of self-compassion. Child y Youth Care Forum, 45(4), 571-586. http://dx.doi.org/10.1007/s10566-016-9346-1

Zeller, M., Yuval, K., Nitzan-Assayag, Y., \& Bernstein, A. (2015). Self-compassion in recovery following potentially traumatic stress: Longitudinal study of at-risk youth. Journal of Abnormal Child Psychology, 43(4), 645-653. http://dx.doi. org/10.1007/s10802-014-9937-y 
